THE ASTROPHYSICAL JOURNAL LETTERS, IN PRESS

Preprint typeset using LATEX style emulateapj v. 08/13/06

\title{
XMM-NEWTON OBSERVATIONS OF CXOU J010043.1-721134: THE FIRST DEEP LOOK AT THE SOFT X-RAY EMISSION OF A MAGNETAR ${ }^{1}$
}

\author{
A. Tiengo, P. Esposito ${ }^{2}$, And S. Mereghetti \\ INAF - Istituto di Astrofisica Spaziale e Fisica Cosmica Milano, \\ Via Edoardo Bassini 15, 20133 Milano, Italy \\ Received 2008 February 11; accepted 2008 May 14
}

\begin{abstract}
We present the analysis of six XMM-Newton observations of the Anomalous X-ray Pulsar CXOU J010043.1721134, the magnetar candidate characterized by the lowest interstellar absorption. In contrast with all the other magnetar candidates, its X-ray spectrum cannot be fit by an absorbed power-law plus blackbody model. The sum of two (absorbed) blackbody components with $k T_{1}=0.30 \pm 0.02 \mathrm{keV}$ and $k T_{2}=0.7 \pm 0.1 \mathrm{keV}$ gives an acceptable fit, and the radii of the corresponding blackbody emission regions are $R_{\mathrm{BB} 1}^{\infty}=12.1_{-1.4}^{+2.1} \mathrm{~km}$ and $R_{\mathrm{BB} 2}^{\infty}=1.7_{-0.5}^{+0.6} \mathrm{~km}$. The former value is consistent with emission from a large fraction of a neutron star surface and, given the well known distance of CXOU J010043.1-721134, that is located in the Small Magellanic Cloud, it provides the most constraining lower limit to a magnetar radius ever obtained. A more physical model, where resonant cyclotron scattering in the magnetar magnetosphere is taken into account, has also been successfully applied to this source.
\end{abstract}

Subject headings: pulsars: individual (CXOU J010043.1-721134) - stars: neutron

\section{INTRODUCTION}

The Anomalous X-ray Pulsars (AXPs; see Mereghetti 2008 , for a recent review) were initially identified as a subclass of accreting $\mathrm{X}$-ray pulsars. Their much softer X-ray spectrum and the lack of a bright optical counterpart distinguished them from the vast majority of X-ray pulsars, which are neutron stars accreting in high mass $\mathrm{X}$-ray binary systems. AXPs have rotation periods of several seconds and show a secular spin-down on timescales of $10^{3}-10^{5}$ years, but their rotational energy loss is smaller than their X-ray luminosity, excluding the possibility that they are rotation-powered, like radio pulsars. It is generally believed that the AXPs, as well as another small class of high-energy sources with similar properties, the Soft Gamma-ray Repeaters (SGRs), are magnetars, i.e. neutron stars powered by their extremely high magnetic field ( $\sim 10^{15} \mathrm{G}$; Duncan \& Thompson 1992; Thompson \& Duncan 1996).

The soft X-ray (1-10 keV) spectra of magnetars cannot be adequately fit with single component models whenever data with good count statistics are available. Successful fits are instead obtained by a two component model consisting of a steep power-law (photon index 3-4) and a blackbody $(k T \sim 0.5 \mathrm{keV})$. Some attempts have been done, also based on phase-resolved spectroscopy, to attribute the two components to physically distinct processes (e.g. Tiengo et al. 2005), but no particularly compelling interpretations could be obtained. One problem of this model is that it tends to give best-fit values of the interstellar absorption higher than those independently estimated in other ways (e.g. Durant \& van Kerkwijk 2006). Another problem is that the power-law component cannot be extrapolated at lower energies without exceeding the flux of the near infrared (NIR) and optical counterparts (e.g. Hulleman et al. 2004). Drastic, and possibly unphysical,

\footnotetext{
Electronic address: tiengo@iasf-milano.inaf.it

${ }^{1}$ Based on observations obtained with XMM-Newton, an ESA science mission with instruments and contributions directly funded by ESA Member States and NASA.

${ }^{2}$ Università di Pavia, Dipartimento di Fisica Nucleare e Teorica and INFNPavia, via Agostino Bassi 6, 27100 Pavia, Italy
}

cut-offs in the power-law component are required to match the low optical/NIR fluxes.

In some AXPs good spectral fits are obtained with the sum of two blackbody components with different temperatures. Since this model does not suffer of the problems described above, it is usually preferred to the power-law plus blackbody model (e.g. Halpern \& Gotthelf 2005). However, also this model is only phenomenological and it is inadequate to represent the non-thermal phenomena that are expected to occur in the highly magnetized magnetosphere of magnetars (e.g. Lyutikov \& Gavriil 2006). More physical models of the X-ray spectra, including the effects of the strong magnetic field and charged currents, have recently been developed and successfully applied to a sample of magnetar candidates (Fernández \& Thompson 2007; Güver et al. 2007; Rea et al. 2008).

From a purely observational point of view, it has not been possible to discriminate between the different models reproducing the magnetar X-ray spectra. This is mainly due to the low sensitivity of hard $\mathrm{X}$-ray detectors above $\sim 10 \mathrm{keV}$ and to the large uncertainties in the fits introduced by the high interstellar absorption, that severely suppresses the flux below $\sim 1$ $\mathrm{keV}$. Being young neutron stars born from massive progenitors, all the Galactic magnetars are located in highly absorbed regions of the Galactic plane. All of them have column densities $\mathrm{N}_{\mathrm{H}}$ ranging from $\sim 5 \times 10^{21}$ to $\sim 10^{23} \mathrm{~cm}^{-2}$. The two known magnetars in the Magellanic Clouds, being considerably less absorbed, offer the possibility to better constrain the spectra in the low energy range. The study of SGR 0526-66, located in the Large Magellanic Cloud, is complicated by the presence of the surrounding supernova remnant N49, which is particularly bright in soft X-rays (Kulkarni et al. 2003). Here we concentrate therefore on the spectral properties of CXOU J010043.1-721134 (Lamb et al. 2002, 2003; McGarry et al. 2005), the only known AXP in the Small Magellanic Cloud (SMC). 
TABLE 1

LOG OF THE XMM-Newton OBSERVATIONS OF CXOU J010043.1-721134. THE PULSE PERIODS AND Corresponding $1 \sigma$ ERrors Are Also REPORTED.

\begin{tabular}{ccccc}
\hline \hline Obs. & Date & $\begin{array}{c}\text { PN exposure } \\
(\mathrm{ks})\end{array}$ & $\begin{array}{c}\text { MOS exposure } \\
(\mathrm{ks})\end{array}$ & $\begin{array}{c}\text { Period } \\
(\mathrm{s})\end{array}$ \\
\hline A & 17 Oct 2000 & 14 & 20 & $8.019(1)$ \\
B & 20 Nov 2001 & 22 & 27 & $8.0193(9)$ \\
C & 27 Mar 2005 & $\ldots$ & 24 & $8.0215(9)$ \\
D & 27 Nov 2005 & 14 & 17 & $8.022(1)$ \\
E & 29 Nov 2005 & 13 & 16 & $8.022(1)$ \\
F & 11 Dic 2005 & 9 & 16 & $8.022(2)$ \\
\hline
\end{tabular}

${ }^{\mathrm{a}}$ The PN data were not considered, since the PN instrument was operated with the filter wheel in closed position.

\section{OBSERVATIONS AND DATA ANALYSIS}

The field containing CXOUJ010043.1-721134 was observed six times ${ }^{3}$ with the XMM-Newton satellite (see Table 1). Here we report the analysis of the data collected by the EPIC instrument, which is composed by one PN (Strüder et al. 2001) and two MOS X-ray cameras (Turner et al. 2001).

CXOU J010043.1-721134 was not the main target of the observations, but, being at an off-axis angle of $\sim 6^{\prime}$, it was always well inside the field of view of the EPIC instrument ( $\sim 15^{\prime}$ radius). All the observations were performed with the medium optical blocking filter and in Full Frame mode (time resolution of $73 \mathrm{~ms}$ and $2.6 \mathrm{~s}$ for the PN and MOS, respectively), except for the first PN observation, done in Extended Full Frame mode (time resolution of $200 \mathrm{~ms}$ ). All the data were processed using the XMM-Newton Science Analysis Software (SAS version 7.1.0) and the calibration files released in August 2007. The standard pattern selection criteria (patterns 0-4 for PN and 0-12 for MOS) were adopted.

Source spectra were extracted for each observation from circular regions with $25^{\prime \prime}$ radius. The background spectra were extracted from a $94^{\prime \prime} \times 72^{\prime \prime}$ box centered at RA $=$ $01^{\mathrm{h}} 00^{\mathrm{m}} 56.8$, Dec. $=-72^{\circ} 11^{\prime} 33^{\prime \prime}$ and rotated such that it intercepts no CCD gaps in any PN image. Response matrices and ancillary files for each spectrum were produced using the SAS software.

The spectra were fitted to a set of models (power-law, blackbody, blackbody plus power-law, and two blackbodies, all modified by photoelectric absorption) using XSPEC version 11.3.1. The single component models gave only marginally acceptable fits in most observations, while better results were obtained with the two component models. The best-fit parameters of the latter models are reported in Table 2.

In order to check for flux variability, we have also simultaneously fit the five available PN spectra and the two MOS spectra for observation $\mathrm{C}$ with a double blackbody model with all parameters linked to the same value and a variable normalization factor. From this analysis we can exclude significant $(>3 \sigma)$ flux variations larger than $\sim 20 \%$ among the different XMM-Newton observations.

Since no significant differences in the spectral parameters are detected and the calibration of the PN instrument has proven to be very stable throughout the XMM-Newton mission (see, e.g., Kirsch et al. 2005), a cumulative spectrum of

\footnotetext{
3 Only the data of the two first observations have already been published (Lamb et al. 2002; Nazé et al. 2004; Majid et al. 2004; McGarry et al. 2005; Nakagawa et al. 2007); we reanalyzed them using more recent calibration files, in order to consistently compare the results with those of the new observations.
}

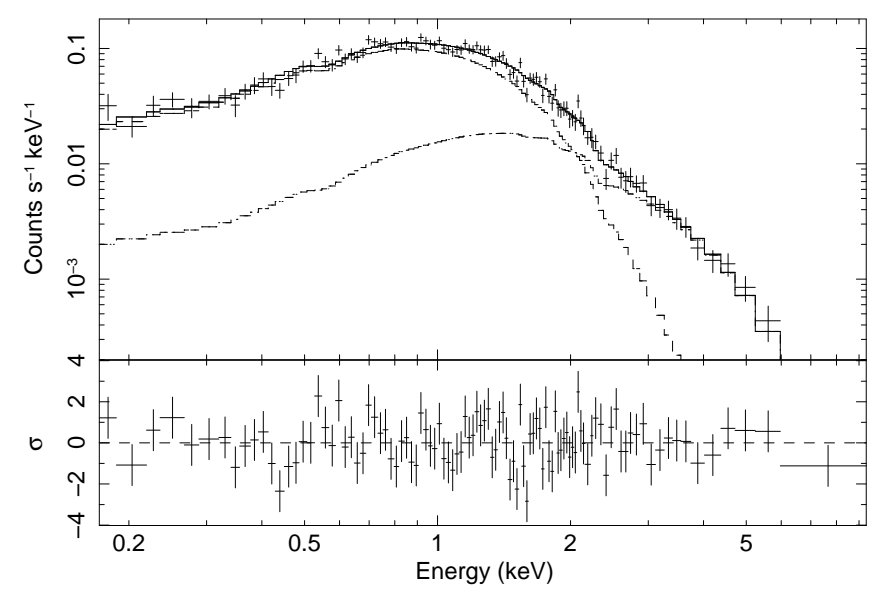

FIG. 1.- Cumulative PN spectrum of CXOU J010043.1-721134 during observations B, D, E, and F. The best-fit double blackbody model is also shown together with the residuals and the separate contribution of the two blackbody components (dotted and dash-dotted lines).

the PN data of observations $\mathrm{B}, \mathrm{D}, \mathrm{E}$, and $\mathrm{F}^{4}$ has also been extracted. The resulting net exposure time is $58 \mathrm{ks}$. Only the double blackbody model gives an acceptable fit to the cumulative spectrum with $k T_{1} \sim 0.3 \mathrm{keV}$ and $k T_{2} \sim 0.7 \mathrm{keV}$ (see Fig. 1 and Table 2). The hydrogen column density is in good agreement with the average value of $\mathrm{N}_{\mathrm{H}}=5.9 \times 10^{20} \mathrm{~cm}^{-2}$ expected towards this region of the SMC (Dickey \& Lockman 1990). The observed flux in the $2-10 \mathrm{keV}$ energy range is $1.4 \times 10^{-13} \mathrm{erg} \mathrm{cm}^{-2} \mathrm{~s}^{-1}$, corresponding to an unabsorbed luminosity of $6.1 \times 10^{34} \mathrm{erg} \mathrm{s}^{-1}$ for a distance of $60 \mathrm{kpc}$. The double blackbody model gave also the lowest $\chi^{2}$ values for the spectra of the single observations, but in those cases it was not the only one compatible with the data. In particular, the power-law plus blackbody model, that is rejected with high confidence by the fit to the cumulative spectrum, gave acceptable fits to all the single spectra.

In addition to the phenomenological models described above, we have also fitted the cumulative spectrum with the magnetar spectral model described in Rea et al. (2008). This model, originally proposed by Lyutikov \& Gavriil (2006), is based on cyclotron resonant scattering of blackbody radiation in a twisted magnetosphere (Thompson et al. 2002). Although the resulting $\chi_{r}^{2}$ (1.20 for 100 degrees of freedom) is slightly worse than for the double blackbody model, the fit is acceptable. The photoelectric absorption $\left(\mathrm{N}_{\mathrm{H}}=5 \pm 1 \times 10^{20}\right.$ $\left.\mathrm{cm}^{-2}\right)$ and blackbody temperature $(k T=0.32 \pm 0.08 \mathrm{keV})$ are consistent with the values derived from the double blackbody fit $\left(\mathrm{N}_{\mathrm{H}}=6.3_{-1.6}^{+2.0} \times 10^{20} \mathrm{~cm}^{-2}\right.$ and $k T=0.30 \pm 0.02 \mathrm{keV}$ for the cooler blackbody). The best-fit values of the other spectral parameters are a resonant scattering optical depth of $\tau_{\text {res }}=1.2 \pm 0.2$ and a particle velocity of $\beta_{T}=0.48 \pm 0.12$. These parameters are in the same range as the ones observed in the other magnetar candidates (Rea et al. 2008). Although a direct information on the size of the emitting region cannot be derived in the current version of this model, an approximate estimate gives a radius similar to the one of the cooler component in the double blackbody model.

By inspecting the residuals from the best-fit models, we found no significant absorption or emission narrow-line features. We computed upper limits on narrow lines' equivalent widths as a function of the assumed line energy and width $\sigma_{E}$. This was done by adding Gaussian components to the

\footnotetext{
4 Observation A has been excluded because it was taken in a different operating mode, while no PN data were available for observation $\mathrm{C}$.
} 
TABLE 2

Summary of the EPIC Spectral Results in the 0.1-10 KEV Energy Range. ERrors Are Given at the $90 \%$ CONFIDENCE LEVEL.

\begin{tabular}{|c|c|c|c|c|c|c|c|c|c|}
\hline Observation & Model $^{\mathrm{a}}$ & $\begin{array}{c}N_{\mathrm{H}}^{\mathrm{b}} \\
\left(10^{20} \mathrm{~cm}^{-2}\right)\end{array}$ & $\Gamma$ & PL norm. ${ }^{c}$ & $\begin{array}{c}k_{B} T_{B B 1} \\
(\mathrm{keV})\end{array}$ & $\begin{array}{c}R_{B B 1}{ }^{\mathrm{d}} \\
(\mathrm{km})\end{array}$ & $\begin{array}{c}k_{B} T_{B B 2} \\
(\mathrm{keV})\end{array}$ & $\begin{array}{c}R_{B B 2}{ }^{\mathrm{d}} \\
(\mathrm{km})\end{array}$ & $\chi_{r}^{2}$ (d.o.f.) \\
\hline \multirow[t]{2}{*}{ A } & $\mathrm{PL}+\mathrm{BB}$ & $4.8_{-2.1}^{+8.4}$ & $1.3_{-1.5}^{+1.1}$ & $0.8_{-0.7}^{+3.9}$ & $0.35_{-0.03}^{+0.02}$ & $9.1_{-0.9}^{+1.4}$ & & & $1.18(83)$ \\
\hline & $\mathrm{BB} 1+\mathrm{BB} 2$ & $8.1_{-4.0}^{+7.9}$ & & & $\begin{array}{l}0.27_{-0.09}^{+0.07} \\
y_{-0.09}\end{array}$ & $12.9_{-3.4}^{+15.7}$ & $0.5_{-0.1}^{+0.3}$ & $3.6_{-2.9}^{+3.5}$ & $1.11(83)$ \\
\hline \multirow[t]{2}{*}{ B } & $\mathrm{PL}+\mathrm{BB}$ & $4.3_{-2.8}^{+3.0}$ & $1.7_{-0.5}^{+0.7}$ & $2.0_{-1.3}^{+5.7}$ & $0.36_{-0.03}^{+0.02}$ & $8.5_{-0.6}^{+1.4}$ & $\cdots$ & & $0.93(115)$ \\
\hline & $\mathrm{BB} 1+\mathrm{BB} 2$ & $4.2_{-2.1}^{+2.8}$ & $\ldots$ & $\ldots$ & $0.31 \pm 0.03$ & $11.0_{-16}^{+2.6}$ & $0.7 \pm 0.1$ & $1.6_{-06}^{+0.9}$ & $0.72(115)$ \\
\hline \multirow[t]{2}{*}{$\mathrm{C}^{\mathrm{e}}$} & $\mathrm{PL}+\mathrm{BB}$ & $7.8_{-7.0}^{+22.1}$ & $\sim 2$ & $<16$ & $0.34_{-0.05}^{+0.04}$ & $9.6_{-2.0}^{+2.80}$ & $\ldots$ & $\ldots$ & $1.16(107)$ \\
\hline & $\mathrm{BB} 1+\mathrm{BB} 2$ & $5.3_{-4.4}^{+21.3}$ & $\ldots$ & $\ldots$ & $<0.39$ & $<11.6$ & $\sim 1$ & $<3.7$ & $1.16(107)$ \\
\hline \multirow[t]{2}{*}{ D } & $\mathrm{PL}+\mathrm{BB}$ & $3.6_{-3.5}^{+6.6}$ & $1.6_{-0.9}^{+0.7}$ & $2.0_{-1.6}^{+5.0}$ & $0.36_{-0.03}^{+0.02}$ & $8.2_{-0.7}^{+1.0}$ & .. & & $1.07(83)$ \\
\hline & $\mathrm{BB} 1+\mathrm{BB} 2$ & $<3.6$ & & & $0.35_{-0.03}^{+0.02}$ & $8.9_{-1.0}^{+0.9}$ & $0.9_{-0.2}^{+0.4}$ & $0.7_{-0.4}^{+0.7}$ & $1.02(83)$ \\
\hline \multirow[t]{2}{*}{ E } & $\mathrm{PL}+\mathrm{BB}$ & $4.2_{-3.2}^{+7.2}$ & $1.4_{-1.2}^{+0.5}$ & $1.5_{-1.3}^{+5.0}$ & $0.37_{-0.03}^{+0.02}$ & $8.3_{-1.0}^{+1.1}$ & -3 & & $0.82(84)$ \\
\hline & $\mathrm{BB} 1+\mathrm{BB} 2$ & $4.4_{-2.5}^{+3.7}$ & & $\ldots$ & $0.33_{-0.06}^{+0.04}$ & $10.1_{-1.7}^{+3.5}$ & $0.8_{-0.3}^{+0.2}$ & $1.2_{-0.7}^{+1.6}$ & $0.75(84)$ \\
\hline \multirow[t]{2}{*}{$\mathrm{F}$} & $\mathrm{PL}+\mathrm{BB}$ & $7.6_{-5.6}^{+32.6}$ & $1.6_{-1.0}^{+2.0}$ & $2.6 \pm 2.1$ & $0.33_{-0.06}^{+0.08}$ & $9.9_{-1.5}^{-2.6}$ & $\ldots$ & $\ldots$ & $1.39(65)$ \\
\hline & $\mathrm{BB} 1+\mathrm{BB} 2$ & $6.6_{-3.4}^{+6.4}$ & $\ldots$ & $\ldots$ & $0.31_{-0.08}^{+0.04}$ & $11.7_{-2.3}^{+5.4}$ & $0.8_{-0.3}^{+0.4}$ & $1.0_{-0.6}^{+2.3}$ & $1.30(65)$ \\
\hline \multirow[t]{2}{*}{$\mathrm{B}+\mathrm{D}+\mathrm{E}+\mathrm{F}^{\mathrm{f}}$} & PL+BB & $9.1_{-3.8}^{+7.9}$ & $2.0_{-0.4}^{+0.5}$ & $3.7_{-1.9}^{+5.5}$ & $0.34 \pm 0.02$ & $9.3_{-0.7}^{+0.9}$ & $\ldots$ & $\ldots$ & $1.75(100)$ \\
\hline & $\mathrm{BB} 1+\mathrm{BB} 2$ & $6.3_{-1.6}^{+2.8}$ & & & $0.30 \pm 0.02$ & $12.1_{-1.4}^{+2.1}$ & $0.68_{-0.07}^{+0.09}$ & $1.7_{-0.5}^{+0.6}$ & $1.14(100)$ \\
\hline
\end{tabular}

${ }^{\mathrm{a}} \mathrm{A}$ free normalization factor has been introduced to account for inaccurate flux reconstruction in the MOS spectra, where, in most cases, the source is located on a CCD gap.

${ }^{\mathrm{b}}$ Assuming photoelectric absorption cross section from Balucinska-Church \& McCammon (1992) and abundances from Anders \& Grevesse (1989).

${ }^{\mathrm{c}}$ In units of $10^{-5} \mathrm{ph} . \mathrm{cm}^{-2} \mathrm{~s}^{-1} \mathrm{keV}^{-1}$, at $1 \mathrm{keV}$.

${ }^{\mathrm{d}}$ Assuming a distance of $60 \mathrm{kpc}$.

eOnly MOS data.

${ }^{f}$ Only PN data.

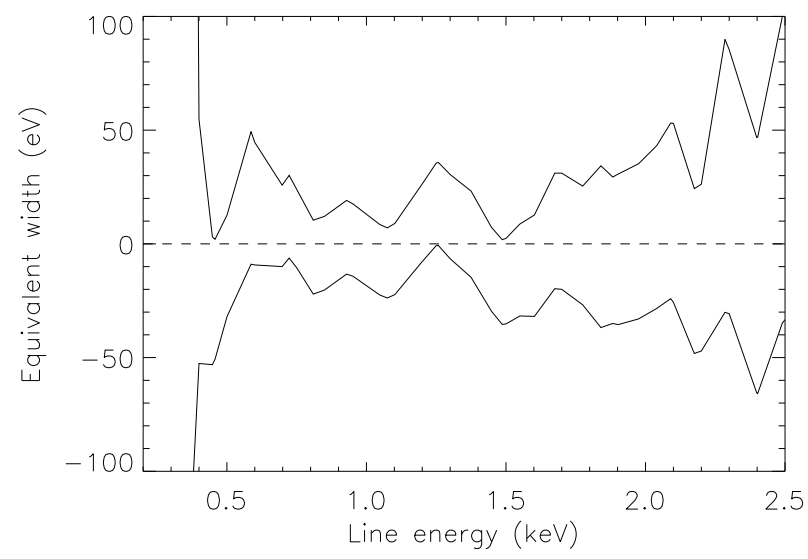

FIG. 2.- Upper limits (at $3 \sigma$ ) to the equivalent width of narrow lines (either in emission or absorption) in the PN cumulative spectrum of CXOU J010043.1-721134 during observations B, D, E, and F.

double blackbody model and computing the allowed range in their normalization. The results for the high-statistics cumulative PN spectrum are summarized in Fig. 2, where the plotted curves represent the $3 \sigma$ upper limits for $\sigma_{E}=0 \mathrm{eV}$.

We performed a timing analysis to measure the source pulse period in each data set. After correcting the photon arrival times to the Solar system barycenter, we derived the best period values based on a $Z_{2}^{2}$ periodogram analysis (Buccheri et al. 1983). The resulting values are indicated in Table 1. Considering also the periods measured by Chandra (McGarry et al. 2005), a linear fit to the ten values yields a period derivative $\dot{P}=(1.9 \pm 0.1) \times 10^{-11} \mathrm{~s} \mathrm{~s}^{-1}\left(\chi_{r}^{2}\right.$ of 1.32 for 8 degrees of freedom).

Since observation D and E were performed only two days apart, we tried to better constrain the spin-down rate through a phase-coherent timing analysis of the two datasets. However, the periods uncertainties during each observation are too large to allow the prediction of the phase of the next observation to better than a pulse cycle.

Many AXPs and SGRs are known to exhibit significant changes in their pulse profiles (e.g. Kaspi 2007; Göğüş et al. 2002). To search for possible pulse shape variations in CXOU J010043.1-721134 as a function of time, we compared the folded light curves using a Kolmogorov-Smirnov test. Taking into account the unknown relative phase alignment, all the light curves are compatible with the same profile. We therefore summed them after appropriate phase shifts 5 . The resulting pulse profiles in the soft $(0.2-1 \mathrm{keV}, S)$ and hard (1-6 keV, H) energy ranges, together with their hardness ratio (computed as $(H-S) /(H+S)$ ), are shown in Fig. 3. This analysis does not show any significant profile changes with energy. The pulsed fraction ${ }^{6}$ in the $0.2-6 \mathrm{keV}$ energy range is $(32 \pm 3) \%$.

\section{DISCUSSION}

The new XMM-Newton observations reported here indicate that CXOU J010043.1-721134 continued to spin-dow at a rate of $\sim 1.9 \times 10^{-11} \mathrm{~s} \mathrm{~s}^{-1}$, consistent with previous results (McGarry et al. 2005). Although the errors on $\mathrm{P}$ are quite large, there is no evidence for major torque changes. Also the pulse profile, spectral shape and flux are consistent with no major changes, confirming that this AXP is one of the most stable members of its class. This characteristic allows us to sum up all the XMM-Newton observations taken with the same instrumental settings.

The cumulative PN spectrum of CXOU J010043.1-721134 cannot be adequately fit by a power-law plus blackbody model. This is the first magnetar for which such a conclu-

\footnotetext{
${ }^{5}$ We selected the shifts that maximized the Kolmogorov-Smirnov statistics comparing subsequent observations.

6 The pulsed fraction is defined as $\left(C_{\max }-C_{\min }\right) /\left(C_{\max }+C_{\min }\right)$, where $C_{\max }$ and $C_{\min }$ are the background-subtracted count rates at the peak and at the minimum.
} 


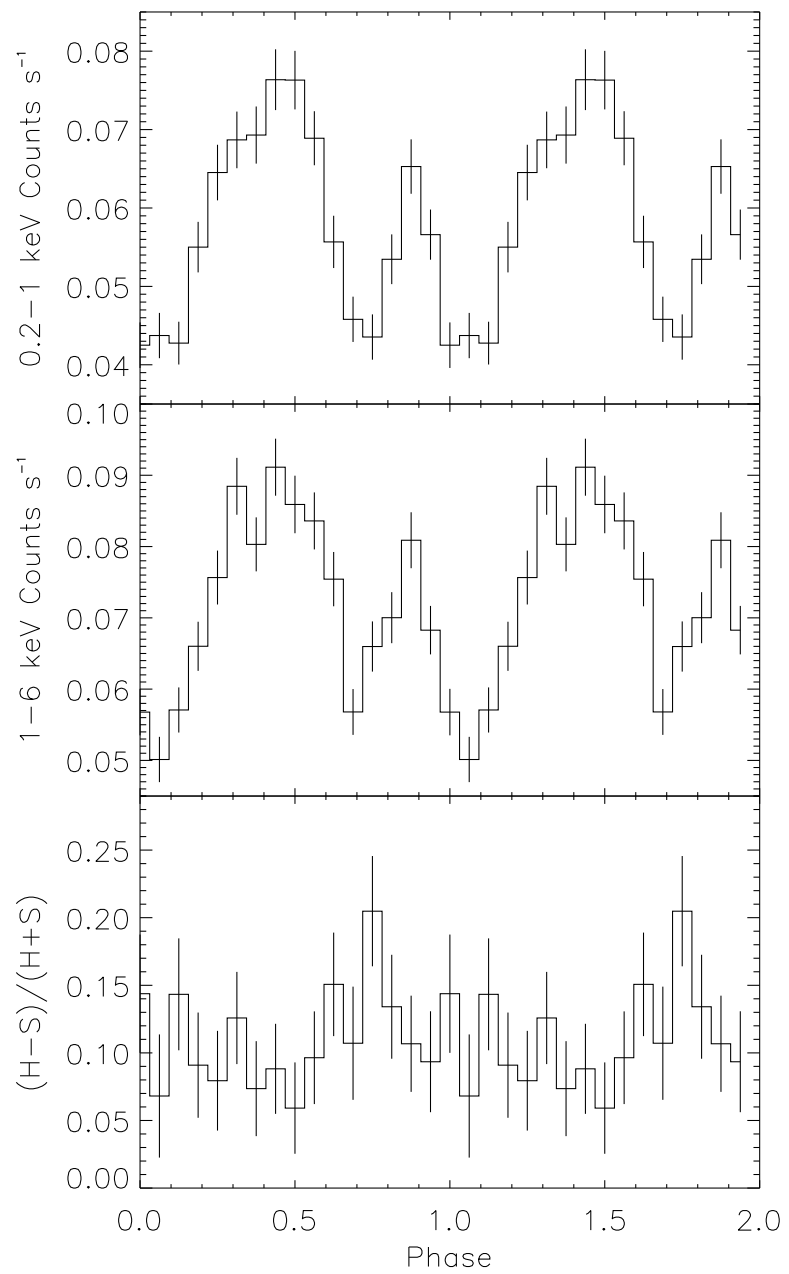

FIG. 3.- Background subtracted pulse profile of CXOU J010043.1721134 in different energy ranges (as indicated in the panels) and corresponding hardness ratio (PN data of observations B, D, E, F).

sion can be derived based only on the X-ray data, thanks to the very low interstellar absorption.

A good fit is instead obtained with a double blackbody model. The known distance of the SMC (60 kpc, Keller \& Wood 2006) allows a precise measure of the size of the emitting region of the two blackbodies. The $\sim 2 \mathrm{~km}$ radius of the region associated to the hotter component is compatible with a hot spot on the neutron star surface. The radius of the emitting region of the cooler blackbody, $12.1_{-1.4}^{+2.1} \mathrm{~km}$, is consistent with a large fraction of the magnetar surface. However, the strong pulsation below $1 \mathrm{keV}$ (see Fig. 3), where this component dominates (see Fig. 1), indicates that it cannot come from the whole neutron star surface. A similarly large blackbody radius $(\sim 10 \mathrm{~km})$ was also derived from the spectrum of the AXP XTE J1810-197 observed by ROSAT before the onset of its outburst in 2003 (Halpern \& Gotthelf 2005) and from phase-resolved spectroscopy of XMM-Newton observations of the same object (Israel et al. 2008) and of the AXP 1E 1048.15937 (Tiengo et al. 2005). However, in these cases, the less accurately known distance and the high interstellar absorption produce large uncertainties on the emitting region size.

Assuming that the thermal photons are produced on the neutron star surface (and not, for instance, in the magnetosphere) and considering that the blackbody is the most efficient thermal emission process at a given temperature, the radius of the region emitting the colder blackbody in
CXOU J010043.1-721134 is a firm lower limit to the radius of the compact object. This limit is not large enough to exclude any of the most popular equations of state for neutron stars, but it is the most constraining lower limit ever obtained for a magnetar.

Magnetar spectra are expected to be more complex than a double blackbody. In fact most magnetar spectra cannot be fitted by such a simple model, that underestimates their emission in the 5-10 keV energy range. In all these cases a power-law tail in hard X-rays $(>20 \mathrm{keV})$ has been detected (see, e.g., Kuiper et al. 2006; Götz et al. 2006; Leyder et al. 2008) and it is likely responsible also for the hard excess below $10 \mathrm{keV}$ (Nakagawa et al. 2007). From a theoretical point of view, the emission expected from a magnetar has two components, with a thermal part directly from the surface, and a non-thermal one due to emission reprocessed in the magnetosphere (Lyutikov \& Gavriil 2006; Fernández \& Thompson 2007). We found that also CXOU J010043.1-721134 can be fit by a model of this kind (Rea et al. 2008).

No compelling detections of spectral features in the persistent X-ray emission of magnetars have been reported so far. XMM-Newton and Chandra observations yielded strong upper limits (equivalent width $\lesssim 10 \mathrm{eV}$ ) for $4 \mathrm{U} 0142+61$ (Juett et al. 2002), 1E 1048.1-5937 (Tiengo et al. 2005), and $1 \mathrm{E} 2259+586$ (Woods et al. 2004) in the $0.7-5 \mathrm{keV}$ range. However, the high interstellar absorption towards these objects causes a series of absorption edges in the observed spectrum at low energies, introducing large systematic uncertainties in the search for features in the intrinsic AXP spectrum. A hint of a spectral feature at $\sim 0.9 \mathrm{keV}$ was noted by Durant \& van Kerkwijk (2006) in the spectrum of the AXP 4U 0142+61, after its deconvolution from interstellar absorption using the edges directly observed in the high resolution X-ray spectra, but also this measure is affected by the poorly constrained abundances of most interstellar elements. As shown in Figure 2, for CXOU J010043.1-721134 we did not find evidence for lines, but, although this source is one of the dimmest AXPs, we could put stringent limits on narrow features in the soft $\mathrm{X}$-ray band, which are virtually independent of the photoelectric absorption model. The dipolar magnetic field derived from the spin-down rate of CXOU J010043.1-721134 is $4 \times 10^{14} \mathrm{G}$, corresponding to a proton cyclotron energy of $\sim 2.5 \mathrm{keV}$; however, a cyclotron line at lower energies is expected if the cyclotron emission or absorption process occurs far from the neutron star surface, while a feature at higher energies is produced if strong multipolar magnetic field components are present. These effects, in addition to other processes that can suppress the spectral features (see, e.g., Ho \& Lai 2003), make the lack of proton cyclotron lines in the $\mathrm{X}$-ray spectra of magnetars compatible with their magnetic fields of $(6-250) \times 10^{13} \mathrm{G}$ (corresponding to proton cyclotron energies of $0.4-15 \mathrm{keV}$ ) derived from their timing properties.

We thank Nanda Rea for fitting the spectrum with the RCS model and useful discussion. This research has been partially supported by the Italian Space Agency. 


\section{REFERENCES}

Anders, E. \& Grevesse, N. 1989, Geochim. Cosmochim. Acta, 53, 197

Balucinska-Church, M. \& McCammon, D. 1992, ApJ, 400, 699

Buccheri, R., et al. 1983, A\&A, 128, 245

Dickey, J. M. \& Lockman, F. J. 1990, ARA\&A, 28, 215

Duncan, R. C. \& Thompson, C. 1992, ApJ, 392, L9

Durant, M. \& van Kerkwijk, M. H. 2006, ApJ, 650, 1082

Fernández, R. \& Thompson, C. 2007, ApJ, 660, 615

Götz, D., Mereghetti, S., Tiengo, A., \& Esposito, P. 2006, A\&A, 449, L31

Göğüş, E., et al. 2002, ApJ, 577, 929

Güver, T., Özel, F., Göğüss, E., \& Kouveliotou, C. 2007, ApJ, 667, L73

Halpern, J. P. \& Gotthelf, E. V. 2005, ApJ, 618, 874

Ho, W. C. G. \& Lai, D. 2003, MNRAS, 338, 233

Hulleman, F., van Kerkwijk, M. H., \& Kulkarni, S. R. 2004, A\&A, 416, 1037

Israel, G. L., et al. 2008, AIP Conf. Proc., 983, 244

Juett, A. M., Marshall, H. L., Chakrabarty, D., \& Schulz, N. S. 2002, ApJ, 568, L31

Kaspi, V. M. 2007, Ap\&SS, 308, 1

Keller, S. C. \& Wood, P. R. 2006, ApJ, 642, 834

Kirsch, M. G., et al. 2005, Proc. SPIE, 5898, 224

Kuiper, L., Hermsen, W., den Hartog, P. R., \& Collmar, W. 2006, ApJ, 645, 556

Kulkarni, S. R., et al. 2003, ApJ, 585, 948

Lamb, R. C., Fox, D. W., Macomb, D. J., \& Prince, T. A. 2002, ApJ, 574 , L29
—. 2003, ApJ, 599, L115

Leyder, J.-C., Walter, R., \& Rauw, G. 2008, A\&A, 477, L29

Lyutikov, M. \& Gavriil, F. P. 2006, MNRAS, 368, 690

Majid, W. A., Lamb, R. C., \& Macomb, D. J. 2004, ApJ, 609, 133

McGarry, M. B., Gaensler, B. M., Ransom, S. M., Kaspi, V. M., \& Veljkovik, S. 2005, ApJ, 627, L137

Mereghetti, S. 2008, preprint (astro-ph/0804.0250)

Nakagawa, Y. E., Yoshida, A., Yamaoka, K., \& Shibazaki, N. 2007, preprint (astro-ph/0710.3816)

Nazé, Y., Manfroid, J., Stevens, I. R., Corcoran, M. F., \& Flores, A. 2004, ApJ, 608, 208

Rea, N., Zane, S., Turolla, R., Lyutikov, M., \& Götz, D. 2008, preprint (astro$\mathrm{ph} / 0802.1923$ )

Strüder, L., et al. 2001, A\&A, 365, L18

Thompson, C. \& Duncan, R. C. 1996, ApJ, 473, 322

Thompson, C., Lyutikov, M., \& Kulkarni, S. R. 2002, ApJ, 574, 332

Tiengo, A., Mereghetti, S., Turolla, R., Zane, S., Rea, N., Stella, L., \& Israel, G. L. 2005, A\&A, 437, 997

Turner, M. J. L., et al. 2001, A\&A, 365, L27

Woods, P. M., et al. 2004, ApJ, 605, 378 